\title{
Rectal Rhabdomyosarcoma
}

National Cancer Institute

\section{Source}

National Cancer Institute. Rectal Rhabdomyosarcoma. NCI Thesaurus. Code C5627.

A malignant mesenchymal tumor with skeletal muscle differentiation affecting the rectum. 